\title{
On a functional equation connected with bi-linear mappings and its Hyers-Ulam stability
}

\section{Krzysztof Ciepliński}

Faculty of Applied Mathematics, AGH University of Science and Technology, Mickiewicza 30, 30-059 Krakow, Poland.

\begin{abstract}
We introduce the notion of a bi-linear mapping which generalizes some known ones, and note that bi-linear mappings satisfy a functional equation. The Hyers-Ulam stability of this equation is studied in Banach, 2-Banach and complete non-Archimedean normed spaces. (C) 2017 All rights reserved.
\end{abstract}

Keywords: Hyers-Ulam stability, functional equation, system of functional equations.

2010 MSC: 39B52, 39B72, 39B82.

\section{Introduction}

Throughout this paper $\mathbb{N}$ stands for the set of all positive integers, $\mathbb{N}_{0}:=\mathbb{N} \cup\{0\}$ and $\mathbb{R}_{+}:=[0, \infty)$.

\subsection{Functional equations}

It is well-known that among functional equations the Cauchy equation

$$
f(x+y)=f(x)+f(y)
$$

and the Jensen equation

$$
f\left(\frac{x+y}{2}\right)=\frac{f(x)+f(y)}{2}
$$

(which is closely connected with the notion of convexity) play a prominent role. A lot of information about them and their applications can be found for instance in [12,13].

Let $X$ and $Y$ be linear spaces over the fields $\mathbb{F}$ and $\mathbb{K}$, respectively. A mapping $f: X \rightarrow Y$ is said to be linear, if

$$
f(a x+b y)=A f(x)+B f(y), \quad x, y \in X
$$

for some $a, b \in \mathbb{F}$ and $A, B \in \mathbb{K}$ (see for example [1]). It is clear that additive and, under the additional assumption that the characteristics of $\mathbb{F}$ and $\mathbb{K}$ are different from 2, Jensen mappings (i.e., solutions of (1.1) and (1.2), respectively) are linear.

Email address: cieplin@agh.edu.pl (Krzysztof Ciepliński)

doi:10.22436/jnsa.010.11.28 
We will say that a function $f: X^{2} \rightarrow Y$ is bi-linear if it is linear in each of its arguments, i.e., $f$ satisfies the following system of functional equations

$$
\begin{cases}f\left(a_{1} x_{1}+a_{2} x_{2}, y\right)=A_{1} f\left(x_{1}, y\right)+A_{2} f\left(x_{2}, y\right), & x_{1}, x_{2}, y \in X \\ f\left(x, b_{1} y_{1}+b_{2} y_{2}\right)=B_{1} f\left(x, y_{1}\right)+B_{2} f\left(x, y_{2}\right), & x, y_{1}, y_{2} \in X\end{cases}
$$

with some $a_{1}, a_{2}, b_{1}, b_{2} \in \mathbb{F}$ and $A_{1}, A_{2}, B_{1}, B_{2} \in \mathbb{K}$.

Let us note that for $a_{1}=a_{2}=b_{1}=b_{2}=1$ and $A_{1}=A_{2}=B_{1}=B_{2}=1$ the above definition leads to the so-called bi-additive mappings (some basic facts on such mappings can be found for instance in [13], where their application to the representation of polynomial functions is also presented); for $a_{1}=$ $a_{2}=b_{1}=b_{2}=\frac{1}{2}$ and $A_{1}=A_{2}=B_{1}=B_{2}=\frac{1}{2}$ we obtain the notion of a bi-Jensen function (which was introduced in 2005 by Prager and Schwaiger (see [17]) with the connection with generalized polynomials; see also [2]); and for $a_{1}=a_{2}=1, b_{1}=b_{2}=\frac{1}{2}$ and $A_{1}=A_{2}=1, B_{1}=B_{2}=\frac{1}{2}$ we get the definition of $a$ Cauchy-Jensen mapping given by Park and Bae in [16].

It is obvious that we have the following.

Remark 1.1. If a function $f: X^{2} \rightarrow Y$ satisfies system (1.3), then

$$
\begin{aligned}
f\left(a_{1} x_{1}+a_{2} x_{2}, b_{1} y_{1}+b_{2} y_{2}\right)= & A_{1} B_{1} f\left(x_{1}, y_{1}\right)+A_{1} B_{2} f\left(x_{1}, y_{2}\right) \\
& +A_{2} B_{1} f\left(x_{2}, y_{1}\right)+A_{2} B_{2} f\left(x_{2}, y_{2}\right)
\end{aligned}
$$

for $x_{1}, x_{2}, y_{1}, y_{2} \in X$

This leads us to the functional equation

$$
\begin{aligned}
f\left(a_{1} x_{1}+a_{2} x_{2}, b_{1} y_{1}+b_{2} y_{2}\right)= & C_{11} f\left(x_{1}, y_{1}\right)+C_{12} f\left(x_{1}, y_{2}\right) \\
& +C_{21} f\left(x_{2}, y_{1}\right)+C_{22} f\left(x_{2}, y_{2}\right),
\end{aligned}
$$

where $a_{1}, a_{2}, b_{1}, b_{2} \in \mathbb{F}$ and $C_{11}, C_{12}, C_{21}, C_{22} \in \mathbb{K}$ are given scalars.

Particular cases of (1.5) are, among others, the following three functional equations:

$$
\begin{array}{r}
f\left(x_{1}+x_{2}, y_{1}+y_{2}\right)=f\left(x_{1}, y_{1}\right)+f\left(x_{1}, y_{2}\right)+f\left(x_{2}, y_{1}\right)+f\left(x_{2}, y_{2}\right), \\
4 f\left(\frac{x_{1}+x_{2}}{2}, \frac{y_{1}+y_{2}}{2}\right)=f\left(x_{1}, y_{1}\right)+f\left(x_{1}, y_{2}\right)+f\left(x_{2}, y_{1}\right)+f\left(x_{2}, y_{2}\right), \\
2 f\left(x_{1}+x_{2}, \frac{y_{1}+y_{2}}{2}\right)=f\left(x_{1}, y_{1}\right)+f\left(x_{1}, y_{2}\right)+f\left(x_{2}, y_{1}\right)+f\left(x_{2}, y_{2}\right) .
\end{array}
$$

Let us point out that these equations characterize bi-additive, bi-Jensen and Cauchy-Jensen mappings, respectively (see $[2,6,16])$.

\subsection{Hyers-Ulam stability}

The following natural question arises in many areas of scientific investigations: what errors we commit replacing functions satisfying some equations only approximately by exact solutions of these equations. Some efficient tools to evaluate these errors can be found in the theory of the Hyers-Ulam stability.

Let us recall that we say that an equation is stable in a class of functions if any function from this class satisfying the equation approximately (in a sense) is near (in a way) to an exact solution of the equation. The problem of stability for homomorphisms of metric groups was formulated by Ulam in 1940, and its solution (for Banach spaces) was published a year later by Hyers.

The method used by Hyers, called the direct method, has been successfully applied for study of the stability of a large variety of equations. Apart from it, there are also several other efficient approaches to the Hyers-Ulam stability, using different tools, for example the fixed point method, the method of invariant means, the method based on sandwich theorems, and the method using the concept of shadowing (see [4] for the details and references). 
In the last few decades, several stability problems of various (functional, differential, difference, integral) equations have been investigated by many mathematicians (see $[3,11]$ for the comprehensive accounts of the subject). In particular, the stability of functional equations (1.6), (1.7) and (1.8) has been studied, among others in $[2,6-8,16-18]$.

In this note, we apply the direct method to prove the Hyers-Ulam stability of (1.5). Moreover, as corollaries from our main results we obtain some stability results on (1.6) and (1.8).

Since the notion of an approximate solution and the idea of nearness of two functions can be understood in various, also nonstandard ways (such non-classical measures of a distance can be introduced, for example, by the notions of a 2-norm and a non-Archimedean norm), we show the stability of (1.5) not only in classical Banach spaces, but also in 2-Banach spaces and complete non-Archimedean normed spaces.

\section{Stability in Banach spaces}

We start with the classical case, i.e., we deal with the stability of functional equation (1.5), and consequently also (1.6) and (1.8), in Banach spaces.

\subsection{Main result}

In this section, we show the Hyers-Ulam stability of (1.5).

Theorem 2.1. Let $\mathrm{Y}$ be a Banach space and $\varepsilon>0$. If $\left|\mathrm{C}_{11}+\mathrm{C}_{12}+\mathrm{C}_{21}+\mathrm{C}_{22}\right|>1$ and $\mathrm{f}: \mathrm{X}^{2} \rightarrow \mathrm{Y}$ is a mapping such that

$$
\left\|f\left(a_{1} x_{1}+a_{2} x_{2}, b_{1} y_{1}+b_{2} y_{2}\right)-C_{11} f\left(x_{1}, y_{1}\right)-C_{12} f\left(x_{1}, y_{2}\right)-C_{21} f\left(x_{2}, y_{1}\right)-C_{22} f\left(x_{2}, y_{2}\right)\right\| \leqslant \varepsilon
$$

for $x_{1}, x_{2}, y_{1}, y_{2} \in X$, then there exists a function $F: X^{2} \rightarrow Y$ satisfying (1.5) and the condition

$$
\|f(x, y)-F(x, y)\| \leqslant \frac{\varepsilon}{\left|C_{11}+C_{12}+C_{21}+C_{22}\right|-1}, \quad x, y \in X
$$

Proof. Set

$$
\mathrm{C}:=\mathrm{C}_{11}+\mathrm{C}_{12}+\mathrm{C}_{21}+\mathrm{C}_{22}, \quad \mathrm{a}:=\mathrm{a}_{1}+\mathrm{a}_{2}, \quad \mathrm{~b}:=\mathrm{b}_{1}+\mathrm{b}_{2} .
$$

Putting $x_{2}=x_{1}$ and $y_{2}=y_{1}$ in (2.1) we get

$$
\left\|f\left(a x_{1}, b y_{1}\right)-C f\left(x_{1}, y_{1}\right)\right\| \leqslant \varepsilon, \quad x_{1}, y_{1} \in X,
$$

whence

$$
\left\|\frac{f\left(a^{n+1} x_{1}, b^{n+1} y_{1}\right)}{C^{n+1}}-\frac{f\left(a^{n} x_{1}, b^{n} y_{1}\right)}{C^{n}}\right\| \leqslant \frac{\varepsilon}{|C|^{n+1}}, \quad x_{1}, y_{1} \in X, \quad n \in \mathbb{N}_{0} .
$$

Consequently, for any $\mathrm{m}, l \in \mathbb{N}_{0}$ with $l<\mathrm{m}$ we have

$$
\left\|\frac{f\left(a^{m} x_{1}, b^{m} y_{1}\right)}{C^{m}}-\frac{f\left(a^{l} x_{1}, b^{l} y_{1}\right)}{C^{l}}\right\| \leqslant \sum_{j=l}^{m-1} \frac{\varepsilon}{|c|^{j+1}}, \quad x_{1}, y_{1} \in X,
$$

and thus for any $x, y \in X,\left(\frac{f\left(a^{n} x, b^{n} y\right)}{C^{n}}\right)_{n \in \mathbb{N}_{0}}$ is a Cauchy sequence. Since $Y$ is a Banach space, this sequence is convergent and we can put

$$
F(x, y):=\lim _{n \rightarrow \infty} \frac{f\left(a^{n} x, b^{n} y\right)}{C^{n}}, \quad x, y \in X
$$

Putting $l=0$ and letting $\mathrm{m} \rightarrow \infty$ in (2.4) we get

$$
\|f(x, y)-F(x, y)\| \leqslant \frac{\varepsilon}{|C|-1}, \quad x, y \in X
$$


i.e., (2.2) holds true.

Next, by (2.1), we obtain

$$
\begin{aligned}
\| \frac{f\left(a^{n}\left(a_{1} x_{1}+a_{2} x_{2}\right), b^{n}\left(b_{1} y_{1}+b_{2} y_{2}\right)\right)}{C^{n}}- & C_{11} \frac{f\left(a^{n} x_{1}, b^{n} y_{1}\right)}{C^{n}}-C_{12} \frac{f\left(a^{n} x_{1}, b^{n} y_{2}\right)}{C^{n}} \\
& -C_{21} \frac{f\left(a^{n} x_{2}, b^{n} y_{1}\right)}{C^{n}}-C_{22} \frac{f\left(a^{n} x_{2}, b^{n} y_{2}\right)}{C^{n}} \| \leqslant \frac{\varepsilon}{|C|^{n}}
\end{aligned}
$$

for $x_{1}, x_{2}, y_{1}, y_{2} \in X$ and $n \in \mathbb{N}_{0}$, whence letting $n \rightarrow \infty$ and using (2.5) we see that

$$
\left\|F\left(a_{1} x_{1}+a_{2} x_{2}, b_{1} y_{1}+b_{2} y_{2}\right)-C_{11} F\left(x_{1}, y_{1}\right)-C_{12} F\left(x_{1}, y_{2}\right)-C_{21} F\left(x_{2}, y_{1}\right)-C_{22} F\left(x_{2}, y_{2}\right)\right\| \leqslant 0
$$

for $x_{1}, x_{2}, y_{1}, y_{2} \in X$, which proves that the function $F$ satisfies (1.5).

\subsection{Some consequences}

Theorem 2.1 with $a_{1}=a_{2}=b_{1}=b_{2}=1$ and $C_{11}=C_{12}=C_{21}=C_{22}=1$ immediately gives the following result on the Hyers-Ulam stability of (1.6).

Corollary 2.2. Let $\mathrm{Y}$ be a Banach space and $\varepsilon>0$. If $\mathrm{f}: \mathrm{X}^{2} \rightarrow \mathrm{Y}$ is a mapping such that

$$
\left\|f\left(x_{1}+x_{2}, y_{1}+y_{2}\right)-f\left(x_{1}, y_{1}\right)-f\left(x_{1}, y_{2}\right)-f\left(x_{2}, y_{1}\right)-f\left(x_{2}, y_{2}\right)\right\| \leqslant \varepsilon
$$

for $x_{1}, x_{2}, y_{1}, y_{2} \in X$, then there exists a function $F: X^{2} \rightarrow Y$ satisfying (1.6) and the condition

$$
\|f(x, y)-F(x, y)\| \leqslant \frac{\varepsilon}{3}, \quad x, y \in X
$$

On the other hand, Theorem 2.1 with $a_{1}=a_{2}=1, b_{1}=b_{2}=\frac{1}{2}$ and $C_{11}=C_{12}=C_{21}=C_{22}=\frac{1}{2}$ also yields the Hyers-Ulam stability of (1.8).

Corollary 2.3. Let $\mathrm{Y}$ be a Banach space and $\varepsilon>0$. If $\mathrm{f}: \mathrm{X}^{2} \rightarrow \mathrm{Y}$ is a mapping such that

$$
\left\|f\left(x_{1}+x_{2}, \frac{y_{1}+y_{2}}{2}\right)-\frac{1}{2} f\left(x_{1}, y_{1}\right)-\frac{1}{2} f\left(x_{1}, y_{2}\right)-\frac{1}{2} f\left(x_{2}, y_{1}\right)-\frac{1}{2} f\left(x_{2}, y_{2}\right)\right\| \leqslant \varepsilon
$$

for $x_{1}, x_{2}, y_{1}, y_{2} \in X$, then there exists a function $F: X^{2} \rightarrow Y$ satisfying (1.8) and the condition

$$
\|f(x, y)-F(x, y)\| \leqslant \varepsilon, \quad x, y \in X .
$$

\section{Stability in 2-Banach spaces}

Recall that the concept of linear 2-normed space was introduced by Gähler in 1964, and it seems that the first work on the Hyers-Ulam stability of functional equations in complete 2-normed spaces (i.e., 2Banach spaces) is [10]. After it some papers (see for instance $[5,8,15]$ ) on the stability of other equations in such spaces have been published.

\subsection{Preliminaries}

Let us recall (see for instance [9]) that by a linear 2-normed space we mean a pair $(X,\|\cdot, \cdot\|)$ such that $X$ is an at least two-dimensional real linear space and $\|\cdot, \cdot\|: X \times X \rightarrow \mathbb{R}_{+}$is a function satisfying the following conditions:

$$
\begin{aligned}
\|x, y\| & =0 \text { if and only if } x \text { and } y \text { are linearly dependent, } \\
\|x, y\| & =\|y, x\|, \quad x, y \in X \\
\|x, y+z\| & \leqslant\|x, y\|+\|x, z\|, \quad x, y, z \in X \\
\|\alpha x, y\| & =|\alpha|\|x, y\|, \quad \alpha \in \mathbb{R}, x, y \in X
\end{aligned}
$$


A sequence $\left(x_{n}\right)_{n \in \mathbb{N}}$ of elements of a linear 2-normed space $X$ is called a Cauchy sequence if there are linearly independent $y, z \in X$ such that

$$
\lim _{n, m \rightarrow \infty}\left\|x_{n}-x_{m}, y\right\|=0=\lim _{n, m \rightarrow \infty}\left\|x_{n}-x_{m}, z\right\|,
$$

whereas $\left(x_{n}\right)_{n \in \mathbb{N}}$ is said to be convergent if there exists an $x \in X$ (called a limit of this sequence and denoted by $\lim _{n \rightarrow \infty} x_{n}$ ) with

$$
\lim _{n \rightarrow \infty}\left\|x_{n}-x, y\right\|=0, \quad y \in X
$$

A linear 2-normed space in which every Cauchy sequence is convergent is called a 2-Banach space.

Let us also mention that in linear 2-normed spaces every convergent sequence has exactly one limit and the standard properties of the limit of a sum and a scalar product are valid.

Finally, recall two lemmas from [15].

Lemma 3.1. If $X$ is a linear 2-normed space, $x \in X$ and

$$
\|x, y\|=0, \quad y \in X,
$$

then $x=0$.

Lemma 3.2. If $X$ is a linear 2-normed space and $\left(x_{n}\right)_{n \in \mathbb{N}}$ is a convergent sequence of elements of $X$, then

$$
\lim _{n \rightarrow \infty}\left\|x_{n}, y\right\|=\left\|\lim _{n \rightarrow \infty} x_{n}, y\right\|, \quad y \in X .
$$

\subsection{Main result}

Now, we show the Hyers-Ulam stability of (1.5) in 2-Banach spaces.

Theorem 3.3. Let $\mathrm{Y}$ be a 2-Banach space and $\varepsilon>0$. If $\left|\mathrm{C}_{11}+\mathrm{C}_{12}+\mathrm{C}_{21}+\mathrm{C}_{22}\right|>1$ and $\mathrm{f}: \mathrm{X}^{2} \rightarrow \mathrm{Y}$ is a mapping such that

$$
\left\|f\left(a_{1} x_{1}+a_{2} x_{2}, b_{1} y_{1}+b_{2} y_{2}\right)-C_{11} f\left(x_{1}, y_{1}\right)-C_{12} f\left(x_{1}, y_{2}\right)-C_{21} f\left(x_{2}, y_{1}\right)-C_{22} f\left(x_{2}, y_{2}\right), z\right\| \leqslant \varepsilon
$$

for $x_{1}, x_{2}, y_{1}, y_{2} \in X$ and $z \in Y$, then there exists a function $F: X^{2} \rightarrow Y$ satisfying (1.5) and the condition

$$
\|f(x, y)-F(x, y), z\| \leqslant \frac{\varepsilon}{\left|C_{11}+C_{12}+C_{21}+C_{22}\right|-1}, \quad x, y \in X, z \in Y .
$$

Proof. Let $\mathrm{C}, \mathrm{a}$ and $\mathrm{b}$ be as in the proof of Theorem 2.1. One can show that for any $m, l \in \mathbb{N}_{0}$ with $l<m$ we have

$$
\left\|\frac{f\left(a^{m} x, b^{m} y\right)}{C^{m}}-\frac{f\left(a^{l} x, b^{l} y\right)}{C^{l}}, z\right\| \leqslant \sum_{j=l}^{m-1} \frac{\varepsilon}{|C|^{j+1}}, \quad x, y \in X, z \in Y,
$$

and therefore for any $x, y \in X,\left(\frac{f\left(a^{n} x, b^{n} y\right)}{C^{n}}\right)_{n \in \mathbb{N}_{0}}$ is a Cauchy sequence. Since $Y$ is a 2-Banach space, this sequence is convergent and we can define $\mathrm{F}: \mathrm{X}^{2} \rightarrow \mathrm{Y}$ by (2.5). Putting $\mathrm{l}=0$ and letting $\mathrm{m} \rightarrow \infty$ in (3.3) we get, by Lemma 3.2,

$$
\|f(x, y)-F(x, y), z\| \leqslant \frac{\varepsilon}{|C|-1}, \quad x, y \in X, z \in Y
$$

i.e., (3.2) holds true.

Next, by (3.1), we obtain

$$
\begin{aligned}
\| \frac{f\left(a^{n}\left(a_{1} x_{1}+a_{2} x_{2}\right), b^{n}\left(b_{1} y_{1}+b_{2} y_{2}\right)\right)}{C^{n}}-C_{11} & \frac{f\left(a^{n} x_{1}, b^{n} y_{1}\right)}{C^{n}}-C_{12} \frac{f\left(a^{n} x_{1}, b^{n} y_{2}\right)}{C^{n}} \\
& -C_{21} \frac{f\left(a^{n} x_{2}, b^{n} y_{1}\right)}{C^{n}}-C_{22} \frac{f\left(a^{n} x_{2}, b^{n} y_{2}\right)}{C^{n}}, z \| \leqslant \frac{\varepsilon}{|c|^{n}}
\end{aligned}
$$

for $x_{1}, x_{2}, y_{1}, y_{2} \in X, z \in Y$ and $n \in \mathbb{N}_{0}$, whence letting $n \rightarrow \infty$ and using (2.5) and Lemmas 3.1 and 3.2 we see that the function $F$ satisfies (1.5). 


\subsection{Some consequences}

Theorem 3.3 with $a_{1}=a_{2}=b_{1}=b_{2}=1$ and $C_{11}=C_{12}=C_{21}=C_{22}=1$ immediately gives the following result on the Hyers-Ulam stability of (1.6).

Corollary 3.4. Let $\mathrm{Y}$ be a 2-Banach space and $\varepsilon>0$. If $\mathrm{f}: \mathrm{X}^{2} \rightarrow \mathrm{Y}$ is a mapping such that

$$
\left\|f\left(x_{1}+x_{2}, y_{1}+y_{2}\right)-f\left(x_{1}, y_{1}\right)-f\left(x_{1}, y_{2}\right)-f\left(x_{2}, y_{1}\right)-f\left(x_{2}, y_{2}\right), z\right\| \leqslant \varepsilon
$$

for $\mathrm{x}_{1}, \mathrm{x}_{2}, \mathrm{y}_{1}, \mathrm{y}_{2} \in \mathrm{X}$ and $z \in \mathrm{Y}$, then there exists a function $\mathrm{F}: \mathrm{X}^{2} \rightarrow \mathrm{Y}$ satisfying (1.6) and the condition

$$
\|f(x, y)-F(x, y), z\| \leqslant \frac{\varepsilon}{3}, \quad x, y \in X, z \in Y .
$$

On the other hand, Theorem 3.3 with $a_{1}=a_{2}=1, b_{1}=b_{2}=\frac{1}{2}$ and $C_{11}=C_{12}=C_{21}=C_{22}=\frac{1}{2}$ also yields the Hyers-Ulam stability of (1.8).

Corollary 3.5. Let $\mathrm{Y}$ be a 2-Banach space and $\varepsilon>0$. If $\mathrm{f}: \mathrm{X}^{2} \rightarrow \mathrm{Y}$ is a mapping such that

$$
\left\|f\left(x_{1}+x_{2}, \frac{y_{1}+y_{2}}{2}\right)-\frac{1}{2} f\left(x_{1}, y_{1}\right)-\frac{1}{2} f\left(x_{1}, y_{2}\right)-\frac{1}{2} f\left(x_{2}, y_{1}\right)-\frac{1}{2} f\left(x_{2}, y_{2}\right), z\right\| \leqslant \varepsilon
$$

for $x_{1}, x_{2}, y_{1}, y_{2} \in X$ and $z \in Y$, then there exists a function $F: X^{2} \rightarrow Y$ satisfying (1.8) and the condition

$$
\|f(x, y)-F(x, y), z\| \leqslant \varepsilon, \quad x, y \in X, z \in Y .
$$

\section{Stability in complete non-Archimedean normed spaces}

Although some particular cases were considered earlier (see [3] for details), the first work on the HyersUlam stability of functional equations in complete non-Archimedean normed spaces was [14]. Over the recent years many papers (see, for instance, $[3,7,18]$ and the references given there) on the stability of different equations in such spaces have been published.

In this section, we present a result on the Hyers-Ulam stability of functional equation (1.5) in complete non-Archimedean normed spaces.

\subsection{Preliminaries}

Let us first recall (see for example $[7,14,18]$ ) some basic definitions and facts concerning nonArchimedean normed spaces.

By a non-Archimedean field we mean a field $\mathbb{F}$ equipped with a function (called a valuation) $|\cdot|: \mathbb{F} \rightarrow$ $\mathbb{R}_{+}$for which

$$
\begin{aligned}
|r| & =0 \text { if and only if } r=0, \\
|r s| & =|r||s|, \quad r, s \in \mathbb{F},
\end{aligned}
$$

and

$$
|r+s| \leqslant \max \{|r|,|s|\}, \quad r, s \in \mathbb{F} .
$$

In such fields we have $|1|=|-1|=1$ and $|n| \leqslant 1$ for $n \in \mathbb{N}_{0}$.

In any field $\mathbb{F}$ the function $|\cdot|: \mathbb{F} \rightarrow \mathbb{R}_{+}$given by

$$
|x|:= \begin{cases}0, & x=0 \\ 1, & x \neq 0\end{cases}
$$

is a valuation which is called trivial, but the most important examples of non-Archimedean fields are p-adic numbers. 
Let $X$ be a linear space over a field $\mathbb{F}$ with a non-Archimedean non-trivial valuation $|\cdot|$. A function $\|\cdot\|: X \rightarrow \mathbb{R}_{+}$is called a non-Archimedean norm provided

$$
\begin{aligned}
\|x\| & =0 \text { if and only if } x=0, \\
\|r x\| & =|r|\|x\|, \quad r \in \mathbb{F}, x \in X
\end{aligned}
$$

and

$$
\|x+y\| \leqslant \max \{\|x\|,\|y\|\}, \quad x, y \in X .
$$

Then $(X,\|\cdot\|)$ (or briefly $X)$ is said to be a non-Archimedean normed space. In any such a space the function $d: X \times X \rightarrow \mathbb{R}_{+}$given by

$$
d(x, y)=\|x-y\|, \quad x, y \in X
$$

is a metric on $X$.

Recall finally that a sequence $\left(x_{n}\right)_{\mathfrak{n} \in \mathbb{N}}$ of elements of a non-Archimedean normed space is Cauchy if and only if the sequence $\left(x_{n+1}-x_{n}\right)_{n \in \mathbb{N}}$ converges to zero, and that the addition, scalar multiplication and non-Archimedean norm are continuous mappings.

\subsection{Main result}

Theorem 4.1. Let $\mathrm{Y}$ be a complete non-Archimedean normed space and $\varepsilon>0$. If $\left|\mathrm{C}_{11}+\mathrm{C}_{12}+\mathrm{C}_{21}+\mathrm{C}_{22}\right|>1$ and $f: X^{2} \rightarrow Y$ is a mapping such that inequality (2.1) holds true for $x_{1}, x_{2}, y_{1}, y_{2} \in X$, then there exists a function $\mathrm{F}: \mathrm{X}^{2} \rightarrow \mathrm{Y}$ satisfying (1.5) and the condition

$$
\|f(x, y)-F(x, y)\| \leqslant \frac{\varepsilon}{\left|C_{11}+C_{12}+C_{21}+C_{22}\right|}, \quad x, y \in X .
$$

Proof. Let $C, a$ and $b$ be as in the proof of Theorem 2.1. Since (2.3) holds, for any $x, y \in X,\left(\frac{f\left(a^{n} x, b^{n} y\right)}{C^{n}}\right)_{n \in \mathbb{N}_{0}}$ is a Cauchy sequence, and thus it is convergent. Therefore, we can define $F: X^{2} \rightarrow Y$ by (2.5).

Next, by induction, we show that

$$
\left\|\frac{f\left(a^{n} x, b^{n} y\right)}{C^{n}}-f(x, y)\right\| \leqslant \frac{\varepsilon}{|C|}, \quad x, y \in X, n \in \mathbb{N},
$$

whence letting $n \rightarrow \infty$ and using (2.5) we get (4.1).

The rest of the proof runs as in the classical case.

\section{Conclusions}

In the paper, we have shown the classical Hyers-Ulam stability of functional equation (1.5) in Banach, 2-Banach and complete non-Archimedean normed spaces. From this we have derived some stability results on two known equations, i.e., (1.6) and (1.8), which characterize bi-additive and Cauchy-Jensen mappings, respectively.

Let us note that one can also prove the generalized (in the sense of Aoki and Rassias or Bourgin and Găvrută) Hyers-Ulam stability of (1.5) as well as obtain similar results via a fixed point method.

In view of Remark 1.1 every function $f: X^{2} \rightarrow Y$ satisfying system (1.3) is a solution of (1.4). Moreover, as we mentioned in Section 1, in some cases (1.4) is equivalent to system (1.3).

We finish the paper by formulating two natural problems.

Problem 5.1. Find all scalars $a_{1}, a_{2}, b_{1}, b_{2} \in \mathbb{F}$ and $A_{1}, A_{2}, B_{1}, B_{2} \in \mathbb{K}$ for which (1.4) and system (1.3) are equivalent.

Problem 5.2. Find a general solution of functional equation (1.4). 


\section{References}

[1] L. Aiemsomboon, W. Sintunavarat, On generalized hyperstability of a general linear equation, Acta Math. Hungar., 149 (2016), 413-422. 1.1

[2] J.-H. Bae, W.-G. Park, On the solution of a bi-Jensen functional equation and its stability, Bull. Korean Math. Soc., 43 (2006), 499-507. 1.1, 1.1, 1.2

[3] N. Brillouët-Belluot, J. Brzdęk, K. Ciepliński, On some recent developments in Ulam's type stability, Abstr. Appl. Anal., 2012 (2012), 41 pages. 1.2, 4

[4] J. Brzdęk, L. Cădariu, K. Ciepliński, Fixed point theory and the Ulam stability, J. Funct. Spaces, 2014 (2014), 16 pages. 1.2

[5] Y. J. Cho, C. Park, M. Eshaghi Gordji, Approximate additive and quadratic mappings in 2-Banach spaces and related topics, Int. J. Nonlinear. Anal. Appl., 3 (2012), 75-81. 3

[6] K. Ciepliński, Generalized stability of multi-additive mappings, Appl. Math. Lett., 23 (2010), 1291-1294. 1.1, 1.2

[7] K. Ciepliński, Stability of multi-additive mappings in non-Archimedean normed spaces, J. Math. Anal. Appl., 373 (2011), 376-383. 4, 4.1

[8] K. Ciepliński, Approximate multi-additive mappings in 2-Banach spaces, Bull. Iranian Math. Soc., 41 (2015), $785-792$. $1.2,3$

[9] R. W. Freese, Y. J. Cho, Geometry of linear 2-normed spaces, Nova Science Publishers, Inc., Hauppauge, NY, (2001). 3.1

[10] J.-M. Gao, On the stability of the linear mapping in 2-normed spaces, Nonlinear Funct. Anal. Appl., 14 (2009), 801-807. 3

[11] S.-M. Jung, Hyers-Ulam-Rassias stability of functional equations in nonlinear analysis, Springer Optimization and Its Applications, Springer, New York, (2011). 1.2

[12] P. Kannappan, Functional equations and inequalities with applications, Springer Monographs in Mathematics, Springer, New York, (2009). 1.1

[13] M. Kuczma, An introduction to the theory of functional equations and inequalities, Cauchy's equation and Jensen's inequality, Second edition, Edited and with a preface by Attila Gilányi, Birkhäuser Verlag, Basel, (2009). 1.1, 1.1

[14] M. S. Moslehian, T. M. Rassias, Stability of functional equations in non-Archimedean spaces, Appl. Anal. Discrete Math., 1 (2007), 325-334. 4, 4.1

[15] W.-G. Park, Approximate additive mappings in 2-Banach spaces and related topics, J. Math. Anal. Appl., 376 (2011), 193-202. 3, 3.1

[16] W.-G. Park, J.-H. Bae, On a Cauchy-Jensen functional equation and its stability, J. Math. Anal. Appl., 323 (2006), 634-643. 1.1, 1.1, 1.2

[17] W. Prager, J. Schwaiger, Multi-affine and multi-Jensen functions and their connection with generalized polynomials, Aequationes Math., 69 (2005), 41-57. 1.1

[18] T. Z. Xu, Stability of multi-Jensen mappings in non-Archimedean normed spaces, J. Math. Phys., 53 (2012), 9 pages. 1.2, $4,4.1$ 